\title{
Implant Movement Analysis (IMA), A New CT Based Technique for Diagnosis of Aseptic Loosening of Total Knee Arthroplasty
}

ISSN: 2576-8875

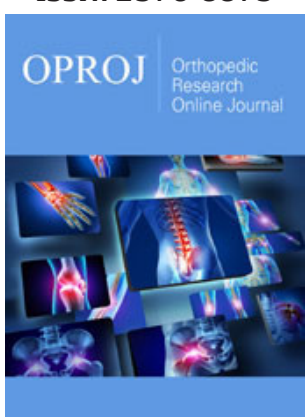

*Corresponding author: Per Wretenberg, Department of Medical Sciences, Örebro University, Sweden

Submission: 侮July 28, 2021

Published: 此 August 03, 2021

Volume 8 - Issue 3

How to cite this article: Per Wretenberg, Simon Tholen, Sofia Carlsson, Henrik Olivecrona. Implant Movement Analysis (IMA), A New CT Based Technique for Diagnosis of Aseptic Loosening of Total Knee Arthroplasty. Ortho Res Online J. 8(3). OPROJ. 000690. 2021.

DOI: 10.31031/OPROJ.2021.08.000690

Copyright@: Per Wretenberg, This article is distributed under the terms of the Creative Commons Attribution 4.0 International License, which permits unrestricted use and redistribution provided that the original author and source are credited.

\author{
Per Wretenberg'1,2*, Simon Tholen ${ }^{3}$, Sofia Carlsson ${ }^{3}$ and Henrik Olivecrona ${ }^{4}$ \\ ${ }^{1}$ Department of Medical Sciences, Örebro University, Sweden \\ ${ }^{2}$ Department of Orthopaedic Surgery, Örebro University Hospital, Sweden \\ ${ }^{3}$ Department of Radiology, Örebro University Hospital, Sweden \\ ${ }^{4}$ Departmentof Molecular Medicine and Surgery, Karolinska Institute, Stockholm, Sweden
}

\begin{abstract}
Background and purpose: Aseptic loosening is the most common indication for revision surgery after Total Knee Arthroplasty (TKA). However, the diagnosis is often difficult from planar radiography as well as other methods, making decision regarding revision surgery problematic. Implant Movement Analysis (IMA) is a newly introduced method of analysing uncertain aseptic loosening of orthopaedic implants. This is the first study where this technique is applied for analysis of potentially loose knee prosthesis. The IMA technique is based on Computer Tomography (CT) with rotational as well as varus and valgus provocation to expose any movement of the implant relatively to the surrounding bone. Our aim was to evaluate the role of IMA as a complementary method to planar radiography and clinical examination in diagnosing aseptic loosening of (TKA).
\end{abstract}

Methods: 40 patients with uncertain aseptic loosening TKA were analysed. All patients had symptoms that could be suspicious for aseptic loosening. Planar radiography was used to detect radiolucent lines. IMA was performed on all patients with rotational as well as varus - valgus provocation.

Results: Of the 40 knees planar radiography showed radiolucent lines in 22 . Out of these 22 knees the IMA analysis showed loosening in 8. IMA showed no loosening in the17 knees without radiolucent lines.

Interpretation: IMA is a dynamic investigation where CT is used during forced provocation of the knee and there by loosening between bone and implant can be more accurate diagnosed. Many suspected TKA loosening's could be ruled out using this method and revision surgery avoided. When planar radiography shows no radiolucent lines the risk of a loose implant is very small.

Keywords: Knee prosthesis; Implant movement analysis; Aseptic loosening; Computed tomography; Radiography

\section{Introduction}

Total knee arthroplasty is one of the most frequent surgical procedures and a very effective treatment option for advanced osteoarthritis [1]. According to the Swedish Knee Arthoplasty Register (SKAR) there were over 17000 primary Total Knee Arthroplasty (TKA) surgeries conducted in 2018 [2]. Generally, the risk for revision surgery after TKA is low [14] defined as when one or more components of the prosthesis is replaced or extracted. Since the number of primary TKA is revision surgery is an increasing problem despite the generally very good results of TKA surgery. Compared with primary TKA revision surgery also has a higher number of perioperative and postoperative complications $[2,5]$.

The two main reason for revision is aseptic loosening and infection [1,5,6]. Aseptic loosening is usually a late-onset complication that can clinically manifest with pain and disability [6,7]. Diagnosing aseptic loosening is primarily done using clinical examinations and planar radiography. On planar radiography loosening can be detected as radiolucent lines (zones) between bone and cement, between cement and implant, or between bone and implant. However, in many cases loosening is less apparent and results from planar 
radiography inconclusive. Therefore, some methods have been developed to try to better diagnose aseptic loosening of total joints. The development has mainly focused on total hip arthroplasty (THA), [8-14], but the methods have also been used for the same purpose for TKA [15-18].

Some of the methods developed to improve imaging analysis of aseptic loosening have been based on converting two-dimensional information from planar radiography into Three-Dimensional (3D) information. One of these methods is called Radiostereometric Analysis (RSA) and is the current gold standard regarding migration measurements $[9,10,14,19,20]$. However, the RSA technique requires the implantation of small metal (tantalum) balls during the primary operation. Therefore, RSA is manly suitable for small groups in research settings. The RSA technique is also difficult to apply on TKA since the femoral component is big and almost surrounds the distal bone of the femur making the tantalum balls hard to detect on planar radiography. To improve imaging diagnostics and find more efficient alternatives, studies have been done using direct 3D imaging diagnostic methods as Magnetic Resonance Imaging (MRI) or Computer Tomography (CT) [11-13]. The focus of this study has been a new CT-based method that recently moved pass the point of experimental testing and was put into clinical use.
The new CT method, Implant Movement Analysis (IMA, Sectra Inc., Linköping) is based on combining two CT volumes with torsion as well as angular loading of the affected knee in forced external and internal rotation as well as varus and valgus acquired in direct sequence. By rotating the knee as well as forcing it in varus and valgus compression this method visualises the implant's movement relative to surrounding bone structures [17,18,21,22]. IMA was developed by Olivecrona et al. [22] at Karolinska Institutet and was in collaboration introduced at Örebro University Hospital, Lindesberg in 2017. It was first used for potentially loose hip implants, but since 2018 also for TKA.

Previously most decisions regarding revision surgery have, in uncertain cases of aseptic loosening, been based on clinical examination, planar radiography and factors including patient age, comorbidity, extent of symptoms and the patient's willingness to go through surgery (Figure 1). Since the introduction of IMA additional imaging information is provided which is believed to improve diagnosing of uncertain cases. This might reduce the number of unnecessary revision surgeries, meaning in cases where loosening was suspected on planar radiography, but the prostheses were in fact in place. The present study is the first study worldwide regarding IMA for TKA.

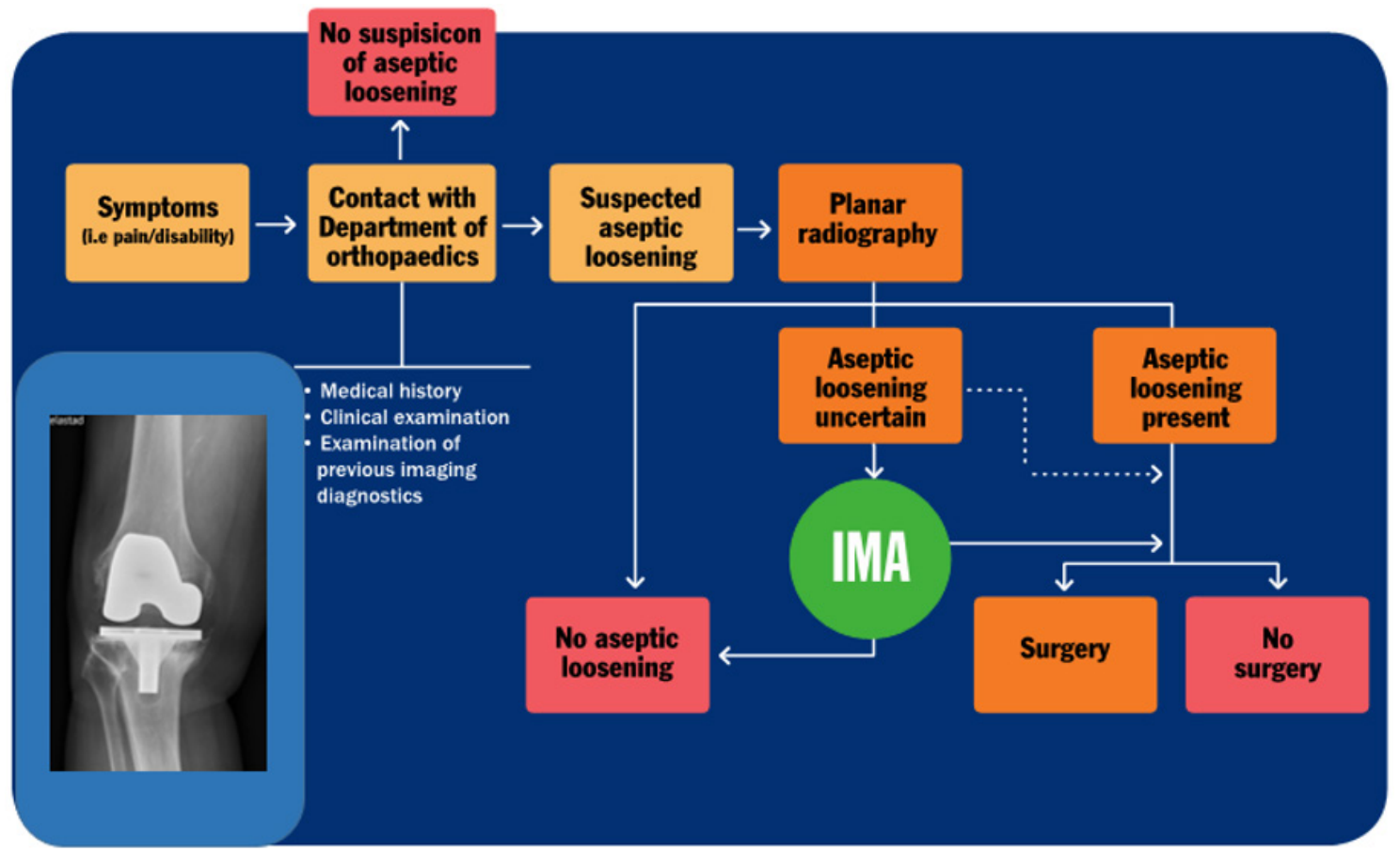

Figure 1: Patient flow chart in cases of suspected aseptic loosening of total knee arthroplasty in Region Örebro County. Dotted line represents flow chart before the introduction of Implant Movement Analysis (IMA).

\section{Methods}

40 patients were admitted to the X-ray department in Lindesberg, Sweden for IMA investigation due to discomfort in their knees that potentially could be because of loosening of their TKA. Radiographic examinations were done according to clinical praxis. All IMA examinations were conducted by two radiographers as described in accordance with instructions given by Henrik Olivecrona, one of the developers of IMA (Figure $2 \& 3$ ). IMA images were analyse by a specially trained radiologist (SK). IMA analysis was not blinded to results from planar radiographic examinations. The software used during IMA examinations is based on methods developed by Olivecrona et al. [21,22]. 

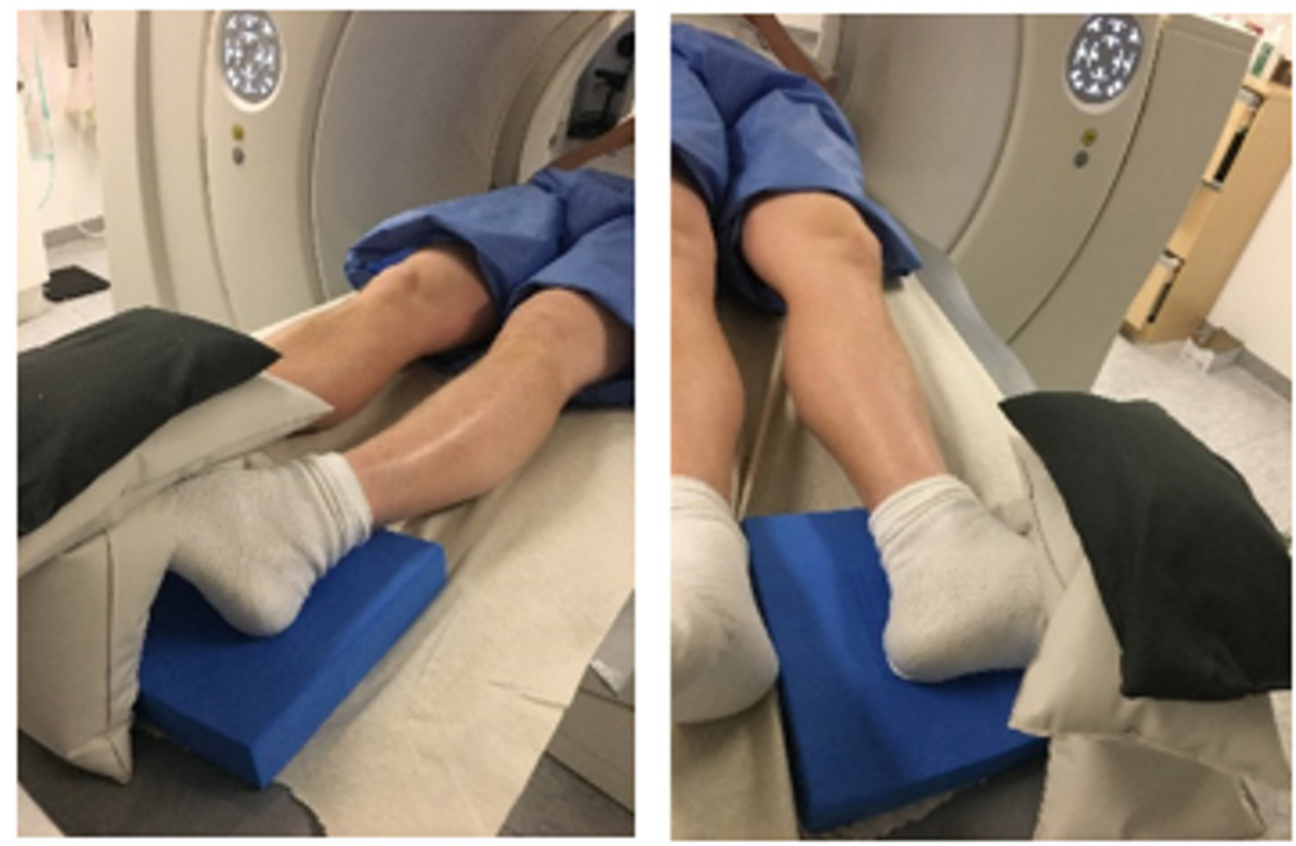

Figure 2: Forced internal and external rotation of the knee.
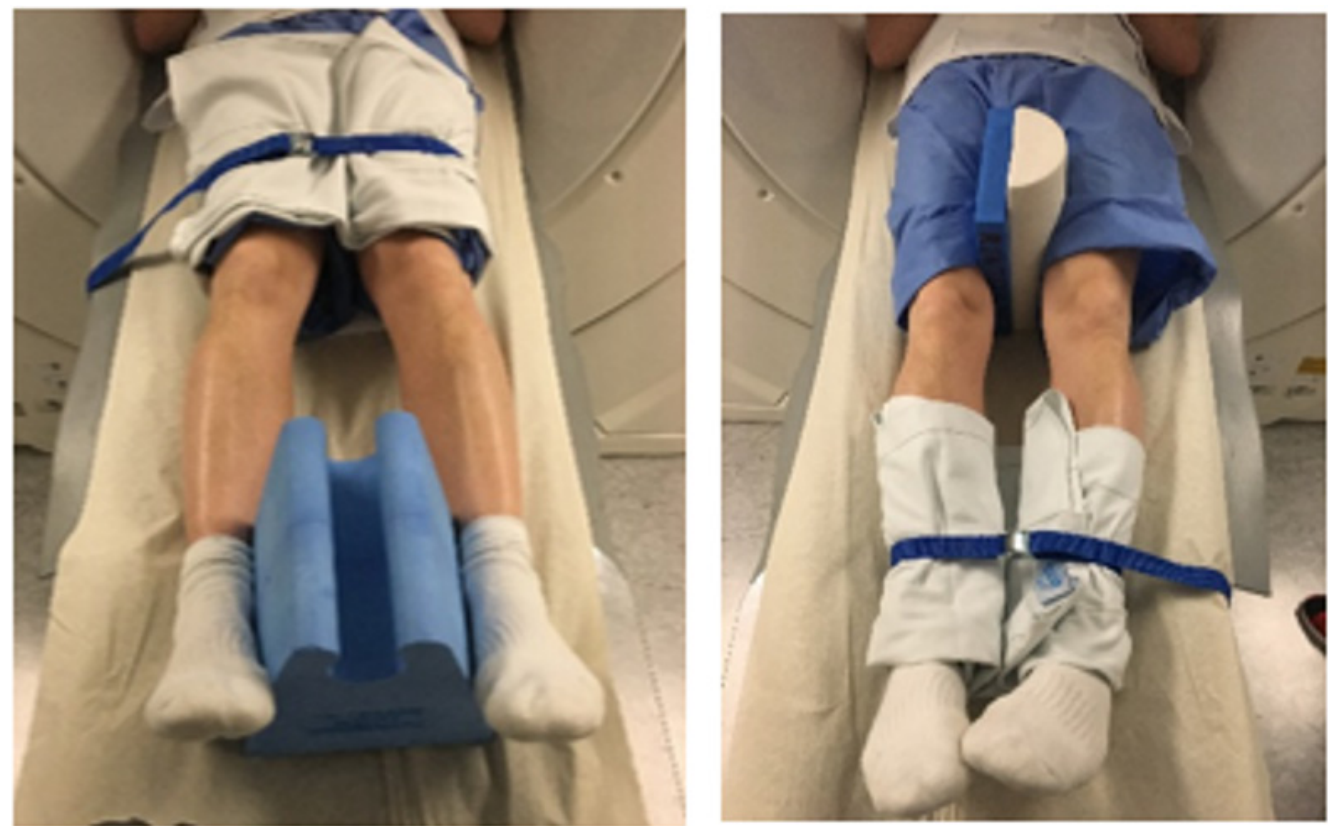

Figure 3: Forced varus- and valgusprovocation of the knee.

Paired examination results from planar radiography and IMA were analysed. In cases were more than one planar radiography examination had been performed previous to IMA, results from the most recent examination were used. Results regarding aseptic loosening were categorized as aseptic loosening; present or not present. These distinctions were primary made by a specially trained radiologist (SK). For study participants who following IMA-examination had surgery, operative reports were also studied. This study was part of a research project approved by the Regional Ethical Review Board in Uppsala (ref. 2019-00117).

\section{Results}

40 consecutive patients were examined between January 2019 December 2020. 23 women and 17 men were included with a mean age at the time of IMA examination of 68 years (range 54 -86). 37 patients were examined after primary TKA and 3 patients after a previous revision. The mean time between the latest TKA surgery and the IMA analysis was 7.5 years (range 2 -21).

Of the 40 TKAs with uncertain loosening 22 were classified as loose on planar radiography mainly based on the presence of 
radiolucent lines. Out of these 22 knees 8 were detected as loose and 14 as stable with use of IMA. Of the 18 knees that were assessed as stable with planar radiography, all were also stable on the IMA analysis (Table 1 \& 2).

Table 1: Findings from planar radiography and Implant Movement Analysis (IMA) in diagnosing aseptic loosening of TKA.

\begin{tabular}{|c|c|c|c|}
\hline Diagnostic Method & Not loose & Loose & n \\
\hline Planar radiography & 18 & 22 & 40 \\
\hline IMA & 32 & 8 & 40 \\
\hline
\end{tabular}

Table 2: Relation between presence of radiolucent lines on planar radiography and a loose implant detected with IMA. If no radiolucent lines are detected the risk of a loose implant is minimal.

\begin{tabular}{|c|c|c|c|}
\hline \multirow{2}{*}{$\begin{array}{c}\text { Loose TKA on } \\
\text { IMA }\end{array}$} & & \multicolumn{2}{|c|}{ Radiolucent Lines } \\
\cline { 2 - 4 } & No & No & Yes \\
\cline { 2 - 4 } & Yes & $0 \% \%$ & $30 \%$ \\
\cline { 2 - 4 } & & $0 \%$ & $25 \%$ \\
\hline
\end{tabular}

The 8 patients that were analysed with a loose implant using IMA were operated with revision surgery and loosening of at least one component was confirmed during surgery.

\section{Discussion}

Revision surgery after TKA is difficult and with a high risk of complications $[23,24]$. Therefore, decision regarding revision surgery should be done with care and the preoperative evaluation is of great importance. When a patient complains about pain and discomfort some years after TKA the reasons for this can be of many different kind [5]. It is important to try to diagnose the main reason to provide the best possible treatment. One common reason for late onset problems after TKA is loosening of the implant and if the prosthesis is loose usually revision surgery is necessary. However, in many cases the diagnosis of aseptic loosening is difficult because the evaluation methods, including planar radiography, is not very accurate. It has recently been shown that young patients have a higher risk of revision [25]. In many cases the revision is of cause needed but there is also a risk that some young people are revised due to presumed loosening that is actually not true.

The aim of this study was that for the first time evaluate Implant Movement Analysis (IMA) as a complementary method to planar radiography and clinical examination in the clinical routine of diagnosing aseptic loosening of TKA. With this technique where CT scan is done during four different sets of forced provocations movement between bone and implant can be visualized on the screen (Figure 4), making the radiographically diagnosis much more accurate than by just watching planar radiographs. Our results indicate that IMA provides very valuable information in the decision-making regarding revision surgery [26,27].

Out of our 40 patients 22 had radiolucent lines on planar radiography to an extent that the radiologist assessed the implant as loose. Out of these 22 only 8 showed a detectable motion between bone and implant with use of IMA. This means that less than half of the patients with loose implants on conventional X-ray proved to be loose during forced provocation. It is well known that revision for pain and discomfort in a TKA knee where the implant is not loose has bad clinical outcome $[5,6]$. To avoid unnecessary revisions is thereby of great importance. Of course, the implant can get loose over time even after the IMA evaluation and follow up is needed. Our preliminary experience is however that many of the patients got satisfied with the message that their prosthesis was stable and became less worried which was beneficial for their function. This means that IMA examination can also be used for patients with high degree of anxiety because of pain and discomfort related to the knee even if the suspicion of a loose implant is low. When the patient receives the information that the TKA is well fixed and that nothing is mechanically wrong, this might lead to an improvement based on psychological reasons.

Another important finding in the present study is that out of the 18 knees without radiolucent lines no proved to be loose. This means that if there are no zones detectable on X-ray the risk of loosening of the implant is very small. To our knowledge this is the first study that has been able to verify this finding.

A limitation in our study is that this is the first study on the use of IMA for diagnosing TKA loosening. This means that there is no possibility to be absolutely sure that the results are correct. We know that of the 8 cases where IMA indicated loosening all were also loose at surgery. This means that the positive predictive value is $100 \%$. The problem is of course on the 32 knees where IMA showed no loosening, how can we know that this result actually is true? The answer is that we cannot. Our plan is to follow these patients over time and to perform a new evaluation 2 years after IMA evaluation with clinical and radiological examination to get an indication of the accuracy of the IMA results. We believe this is the only option to get closer to the true result. However, as the visual presentation of the IMA results are strong and the basic techniques for CT provocation has been developed over many years, we believe that the advantages of starting using IMA at this point in clinical use is great. This is also the only way to gather more information of the method and its value. We can see no disadvantages of introducing this method as a complement to the quite poor evaluation methods available today. The CT is of low-dose type and the radiation in level with a planar radiography and the procedure not to complicated.

The idea of using CT with provocation is not new. Berger et al. [23] and Reinus et al. [24] have been using this kind of technique to evaluate loosening of femoral stems in hip arthroplasty with good results. So, in line with previous findings, we believe that the future potential of IMA is very promising. In conclusion this is the first study of a new standardized technique to evaluation of potentially loose TKA implants. Our short-term experience is promising, and we can see no disadvantages in starting to use IMA as a complement to the methods used today. 


\section{References}

1. Carr AJ, Robertsson O, Graves S, Price AJ, Arden NK, et al. (2012) Knee replacement. Lancet 379(9823): 1331-1340.

2. Registry SKA (2018) The swedish knee arthroplasty register. Annual Report, ISBN 978-91-88017-04-8.

3. (2015) Australian orthopaedic association national joint replacement registry. Annual Report. Adelaide, Australia.

4. Registry NJ (2015) National Joint Registry. 12 ${ }^{\text {th }}$ Annual Report 2015 National Joint Registry for England, Wales, Northern Ireland and the Isle of Man, ISSN 2054-183X.

5. Postler A, Lützner C, Beyer F, Tille E, Lützner J (2018) Analysis of total knee arthroplasty revision causes. BMC Musculoskelet Disord 19(1): 55.

6. Khan M, Osman K, Green G, Haddad FS (2016) The epidemiology of failure in total knee arthroplasty: Avoiding your next revision. Bone Joint J 98-B(1 Suppl A): 105-112.

7. Sadoghi P, Liebensteiner M, Agreiter M, Leithner A, Bohler N, et al. (2012) Revision surgery after total joint arthroplasty: A complicationbased analysis using worldwide arthroplasty registers. J Arthroplast 28(8): 1329-1332.

8. Krismer M, Bauer R, Tschupik J, Mayrhofer P (1995) EBRA: A method to measure migration of acetabular components. J Biomech 28(10): 12251236.

9. Selvik G (1989) Roentgen stereophotogrammetry. A method for the study of the kinematics of the skeletal system. Acta Orthop Scand Suppl 232: 1-51.

10. Nakama GY, Peccin MS, Almeida GJM, Lira Neto ODA, Queiroz AAB, et al. (2021) Cemented, cementless or hybrid fixation options in total knee arthroplasty for osteoarthritis and other non-traumatic diseases. Cochrane Database of Systematic Reviews 10: CD006193.

11. Berkowitz JL, Potter HG (2017) Advanced MRI techniques for the hip joint: Focus on the postoperative hip. AJR Am J Roentgenol 209(3): 534543.

12. Chew CG, Lewis P, Middleton F, van den Wijngaard R, Deshaies A (2010) Radionuclide arthrogram with SPECT/CT for the evaluation of mechanical loosening of hip and knee prostheses. Ann Nucl Med 24(10): 735-743.

13. White LM, Kim JK, Mehta M, Merchant N, Schweitzer ME, et al. (2000) Complications of total hip arthroplasty: MR imaging-initial experience. Radiology 215(1): 254-262.

14. Streit MR, Haeussler D, Bruckner T, Proctor T, Innmann MM, et al. (2016) Early migration predicts aseptic loosening of cementless femoral stems: A long-term study. Clin Orthop Relat Res 474(7): 1697-1706.
15. Scheerlinck T, Polfliet M, Deklerck R, Van Gompel G, Buls N, et al. (2016) Development and validation of an automated and marker-free CTbased spatial Analysis Method (CTSA) for assessment of femoral hip implant migration: In vitro accuracy and precision comparable to that of Radiostereometric Analysis (RSA). Acta Orthop 87(2):139-145.

16. Jedenmalm A, Noz ME, Olivecrona H, Olivecrona L, Stark A (2008) A new approach for assessment of wear in metal-backed acetabular cups using computed tomography: A phantom study with retrievals. Acta Orthop 79(2): 218-224.

17. Brodén C, Olivecrona H, Maguire GQ, Noz ME, Zeleznik MP, et al. (2016) Accuracy and precision of three-dimensional low dose CT compared to standard rsa in acetabular cups: An experimental study. Biomed Res Int.

18. Olivecrona L, Crafoord J, Olivecrona H, Noz ME, Maguire GQ, et al. (2002) Acetabular component migration in total hip arthroplasty using CT and a semiautomated program for volume merging. Acta Radiol 43(5): 517527.

19. Wilkinson JM, Hamer AJ, Elson RA, Stockley I, Eastell R (2002) Precision of EBRA-Digital software for monitoring implant migration after total hip arthroplasty. J Arthroplasty 17(7): 910-916.

20. Jacobsen A, Seehaus F, Hong Y, Cao H, Schuh A, et al. (2018) Modelbased roentgen stereophotogrammetric analysis using elementary geometrical shape models: 10 years results of an uncemented acetabular cup component. BMC Musculoskelet Disord 19(1): 335.

21. Olivecrona L (2010) A new computed tomography method for evaluation of orthopedic implants: Applied to the acetabular cup, Stockholm: Karolinska Institutet, Sweden.

22. Olivecrona H, Olivecrona L, Weidenhielm L, Noz ME, Kardos J, et al. (2008) A new technique for diagnosis of acetabular cup loosening using computed tomography: Preliminary experience in 10 patients. Acta Orthop 79(3): 346-353.

23. Berger R, Fletcher F, Donaldson T, Wasielewski R, Peterson M, et al. (1996) Dynamic test to diagnose loose uncemented femoral total hip components. Clin Orthop Relat Res 330:115-123.

24. Reinus WR, Merkel KC, Gilden JJ, Berger KL (1996) Evaluation of femoral prosthetic loosening using CT imaging. AJR Am J Roentgenol 166(6): 1439-1442.

25. Lei PF, Hu RY, Hu YH (2019) Bone defects in revision total knee arthroplasty and management. Orthop Surg 11(1):15-24.

26. Fraser JF, Werner S, Jacofsky DJ (2015) Wear and loosening in total knee arthroplasty: A quick review. J Knee Surg 28(2): 139-144.

27. Bayliss LE, Culliford D, Monk AP, Glyn-Jones S, Prieto-Alhambra D, et al. (2017) The effect of patient age at intervention on risk of implant revision after total replacement of the hip or knee: A population-based cohort study. Lancet 389(10077): 1424-1430. 